\title{
P-13: Photosensitivity of Amorphous IGZO TFTs for Active-Matrix Flat-Panel Displays
}

\author{
Chiao-Shun Chuang ${ }^{a, c}$, Tze-Ching Fung ${ }^{a}$, Barry G. Mullins ${ }^{a}$, Kenji Nomura ${ }^{b}$, \\ Toshio Kamiya ${ }^{b}$, Han-Ping David Shieh ${ }^{c}$, Hideo Hosono ${ }^{b}$ and Jerzy Kanicki ${ }^{a}$ \\ ${ }^{a}$ Dept. of Electrical Engineering and Computer Science, University of Michigan, Ann Arbor, \\ Michigan, 48105 USA \\ ${ }^{b}$ ERATO-SORST, JST, in Frontier Collaborative Research Center / Materials and Structures Laboratory, \\ Mail Box R3-1, Tokyo Institute of Technology 4259 Nagatsuta, Midori-ku, Yokohama 226-8503, Japan \\ ${ }^{\mathrm{c}}$ Dept. of Photonics \& Display Institute, National Chiao Tung University, Hsinchu, Taiwan, 30010 R.O.C.
}

\begin{abstract}
We studied the optical and electrical properties of the amorphous indium gallium zinc oxide thin-film transistors (a-IGZO TFTs). To develop a-IGZO density-of-states model, intrinsic a-IGZO optical properties such as optical band gap and Urbach energy, and TFT characteristics under illumination are investigated. During the aIGZO TFTs illumination with the wavelengths ranging from 460 to $660 \mathrm{~nm}$, the off-state drain current only slightly increases while a large increase was observed for the wavelength below $400 \mathrm{~nm}$. Threshold voltage and subthreshold swing are also only slightly modified between 460 to $660 \mathrm{~nm}$, while field-effect mobility is almost unchanged in the investigated photon energy range. The observed results are consistent with the a-IGZO optical energy band gap of about $3.05 \mathrm{eV}$. This study suggest that the a-IGZO TFTs are light sensitive above $3.0 \mathrm{eV}$ and photogenerated electrons are more mobile than holes within device channel region.
\end{abstract}

\section{Introduction}

Today, conventional active-matrix (AM) flat panel displays (FPDs) are based on amorphous or polycrystalline silicon thin-film transistor (TFT) technology. Limitations of amorphous silicon (a$\mathrm{Si}: \mathrm{H})$ include visible light sensitivity and a low field-effect mobility, which reduce the pixel aperture ratio and driving ability for some applications. Although polycrystalline silicon TFTs have a larger field-effect mobility, their uniformity over large area might not acceptable for high yield manufacturing. Over the last several years, there has been great interest in thin-film transistors made of transparent oxide semiconductors [1-3]. This is mainly due to metal oxide semiconductor thin-film transistors unique advantages, such as visible light transparency, large-area uniform deposition at low temperature, and high carrier mobility. However, conventional metal oxide semiconductors such as zinc oxide $(\mathrm{ZnO})$ are polycrystalline in nature, even at room temperature. The grain boundaries of such metal oxides could affect device properties, uniformity and stability over large areas. To overcome this issue, a new ternary oxide material comprised of $\mathrm{In}, \mathrm{Ga}, \mathrm{Zn}$ and $\mathrm{O}$ has been proposed for use as the channel layer in TFTs [4]. The amorphous In-Ga-Zn-O (a-IGZO) can more easily form a uniform amorphous phase while still having a high carrier mobility, like most oxide semiconductors. Although several papers have discussed the aIGZO TFT electrical performance and its visible light transparency, a more in-depth photosensitivity study is necessary for implementing this technology in real display or photo-sensor products. In this paper, we report on the optical and electrical properties of amorphous a-IGZO TFTs, which are suitable for active-matrix display and photo-sensor applications.

\section{Experimental}

Fig. 1(a) shows a bottom gate a-IGZO TFT structure used in this study. A heavily doped $\left(\mathrm{n}^{++}\right)$silicon wafer with $100 \mathrm{~nm}$ thermal oxide layer was selected as the gate electrode and insulator, respectively. A 40nm thick a-IGZO (In:Ga:Zn=1:1:1) active layer was deposited on the substrate by pulse-laser deposition (PLD) [5]. A $\operatorname{KrF}$ laser $(\lambda=238 \mathrm{~nm})$ was used and the laser energy density was set at $\sim 2 \mathrm{~J} / \mathrm{cm}^{2}$-pulse. The substrate was placed in an ultra-high vacuum system where the target-to-substrate distance is $3 \mathrm{~cm}$ and the deposition was done in an oxygen atmosphere $(\sim 8 \mathrm{~Pa})$ without any intentional substrate heating. Before the source/drain electrodes deposition, a macro-island was formed by edgedipping/etching of the substrate in $0.1 \mathrm{M} \mathrm{HCl}$ solution. The $50 \mathrm{~nm}$ thick aluminum (Al) source/drain electrodes were deposited through stencil mask openings by thermal evaporation. Finally, the device was thermally annealed in air at $300^{\circ} \mathrm{C}$ for 5 minutes. In order to get a good and uniform photo-response under light illumination, finger-type (interdigitated) source/drain electrodes are chosen as shown in Fig. 1(b).

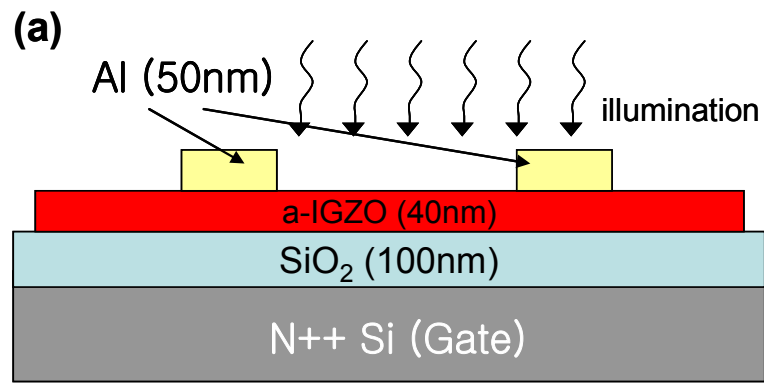

(b)

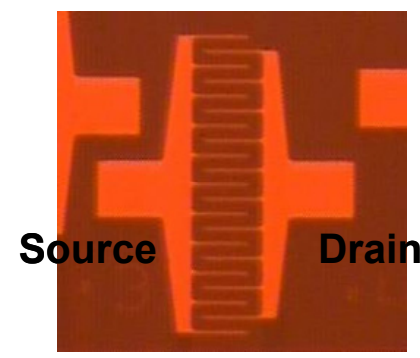

Figure 1. (a) Cross-section and (b) top view of PLD a-IGZO TFT device used in this study. 
We also deposited a-IGZO thin film directly onto quartz substrate to measure its optical properties. The absorption spectrum of a-IGZO thin-film was collected by a Cary 5E UV-VIS spectrometer using polarized light [6]. Quartz substrates were used to minimize the back ground interference during transmittance measurement. Electrical measurement of the a-IGZO TFT were carried out with a probe station system located in a light tight box. The transistor electrical properties were measured by a PC controlled Agilent 4156 Parametric Analyzer. For measuring TFT transfer characteristics, the drain-to-source voltage $\left(\mathrm{V}_{\mathrm{DS}}\right)$ was changed between $0.1 \mathrm{~V}$ and $\mathrm{V}_{\mathrm{GS}}$, and gate-to-source voltage $\left(\mathrm{V}_{\mathrm{GS}}\right)$ was varied from $-10 \mathrm{~V}$ to $20 \mathrm{~V}$. For photoelectric measurement, photo excitation was provided by a He-Xe lamp in combination with narrow band filters and an optical fiber. The monochromic light passed through a fiber cable and probe station microscope, which is used to focus the illumination on the specific device. The wavelengths ranging from 660 to $365 \mathrm{~nm}$ with a constant optical flux of $1 \times 10^{13}$ photons $/ \mathrm{cm}^{2}$-sec incident on the a-IGZO TFT channel were used in this experiment. For each measurement light intensity was calibrated by Oriel 70260 radiant power meter with the photodiode sensor attached. All measurements were done at room temperature in ambient air.

\section{Experimental Results}

\subsection{Optical Properties}

Fig. 2 shows the optical absorption spectrum of the PLD a-IGZO thin films (thickness $=181 \mathrm{~nm}$ ). We assumed parabolic densities of band states within a-IGZO and extracted the optical energy band gap ( $E_{\mathrm{g}}$, also called Tauc gap) by applying the Tauc method [7]:

$$
(\hbar \omega \alpha)^{1 / 2}=B\left(\hbar \omega-E_{g}\right)
$$

where $\hbar \omega$ is the photon energy (E) and $\alpha$ is the absorption coefficient. The Tauc gap $\left(\mathrm{E}_{\mathrm{g}}\right)$ was determined to be $\sim 3.05 \mathrm{eV}$ as shown in Fig. 2. We also observed the exponential energy dependence of the absorption coefficient in the vicinity of $\mathrm{E}_{\mathrm{g}}$ which can be described by [7]:

$$
\alpha \propto \exp \left(\frac{E}{E_{0}}\right)
$$

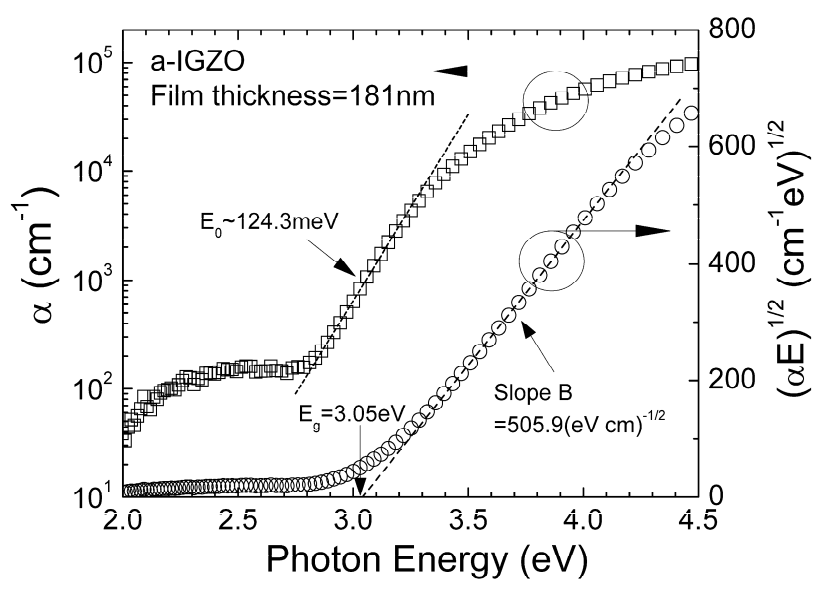

Figure 2. Optical absorption spectrum of PLD a-IGZO thin film. where $\mathrm{E}$ is the photo energy and $\mathrm{E}_{\mathrm{o}}$ is the characteristic slope also frequently called Urbach energy. The Urbach energy is determined to be around $124 \mathrm{meV}$ as indicated in Fig. 2. The Urbach energy is related to the joint density-of-states determined by both conduction and valence band tail states. However, the valence-band-tail can be broader (or shallower) than conduction-band-tail and dominates the optical absorption [8].

As a result, Urbach energy will be fairly close to the valenceband-tail slope value. Such phenomenon had been commonly observed in a-Si:H thin-film; and conduction-band-tail slope is usually about half of the valence-band-tail slope [9]. If we assume a similar situation to occur in a-IGZO, the valence-band-tail slope is approximately $124 \mathrm{meV}$ and corresponding conduction-band-tail slope will be around $62 \mathrm{meV}$. Both values should be confirmed by other methods in near future.

\subsection{TFT Electrical Properties}

\subsubsection{Dark}

Before illumination, we characterized the a-IGZO TFT electrical properties in the dark. Device parameters are extracted by standard MOSFET drain current equations:

$I_{D S}=\mu_{e f f}^{\text {lin }} C_{o x} \frac{W}{L}\left(V_{G S}-V_{t h}-\frac{V_{D S}}{2}\right) V_{D S}$

for linear region and
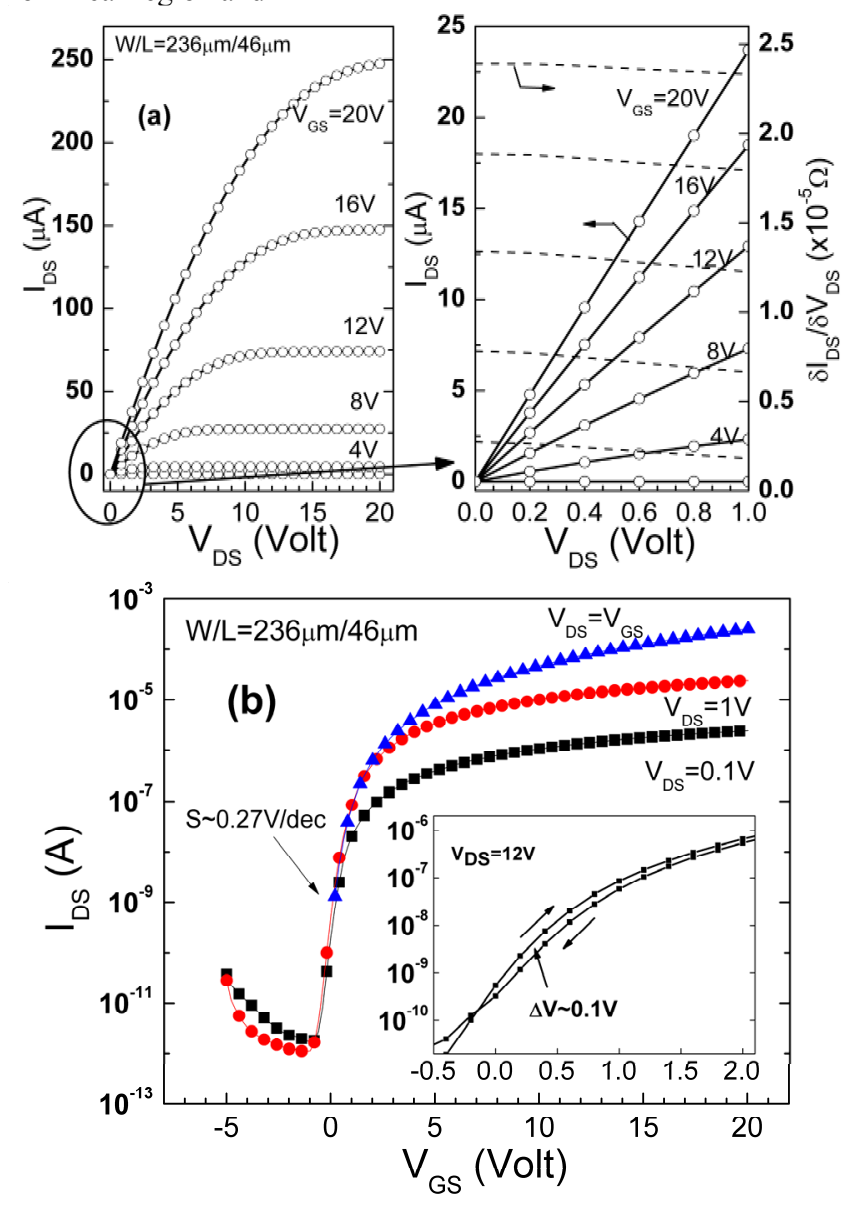

Figure 3. (a) Dark IDS-VDS and (b) IDS-VGS characteristics of PLD a-IGZO TFT. 


$$
I_{D S}=\mu_{e f f}^{s a t} C_{o x} \frac{W}{2 L}\left(V_{G S}-V_{t h}\right)^{2}
$$

for saturation region. $\mathrm{C}_{\mathrm{ox}}$ is the gate insulator capacitance per unit area, $\mu_{e f f}^{\text {lin }}$ and $\mu_{e f f}^{\text {sat }}$ are the effective field-effect mobility, $\mathrm{V}_{\text {th }}$ is the threshold voltage and $\mathrm{W}$ and $\mathrm{L}$ are channel width and length of the TFT device, respectively.

The typical extracted $\mu_{\text {eff }}^{\text {lin }}$ and $\mu_{\text {eff }}^{\text {sat }}$ from the TFT are 7.3 and $8.2 \mathrm{~cm}^{2} / \mathrm{Vs}$, respectively; $\mathrm{V}_{\text {th }}$ value for linear regime is $2.13 \mathrm{~V}$. It should be noted that field-effect-mobility seems to be W/L dependent. We also evaluated the TFT subthreshold swing (S) between off- and on-states, using the following equation:

$$
I_{D S} \propto 10^{V_{G S}} / \mathrm{s}
$$

The TFT exhibits a good subthreshold swing (S) of $0.27 \mathrm{~V} /$ decade

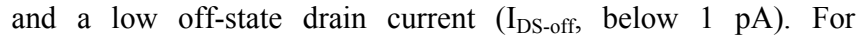
comparison, we conveniently set the on-states drain current ( $\left.\mathrm{I}_{\mathrm{DS}-\mathrm{on}}\right)$ to be the drain current at $\mathrm{V}_{\mathrm{DS}}=\mathrm{V}_{\mathrm{GS}}=20 \mathrm{~V}$. In general, PLD a-IGZO TFT shows good transistor behavior in both saturation regime and linear regimes of operation, Fig. 3(a). As can be seen in Fig. 3(b), the TFT dark on/off current ratio is larger than $10^{8}$ with low hysteresis (inset of Fig. 3(b), $\Delta \mathrm{V} \sim 0.1 \mathrm{~V}$, where $\Delta \mathrm{V}$ is the subthreshold characteristic shift) during measurements.

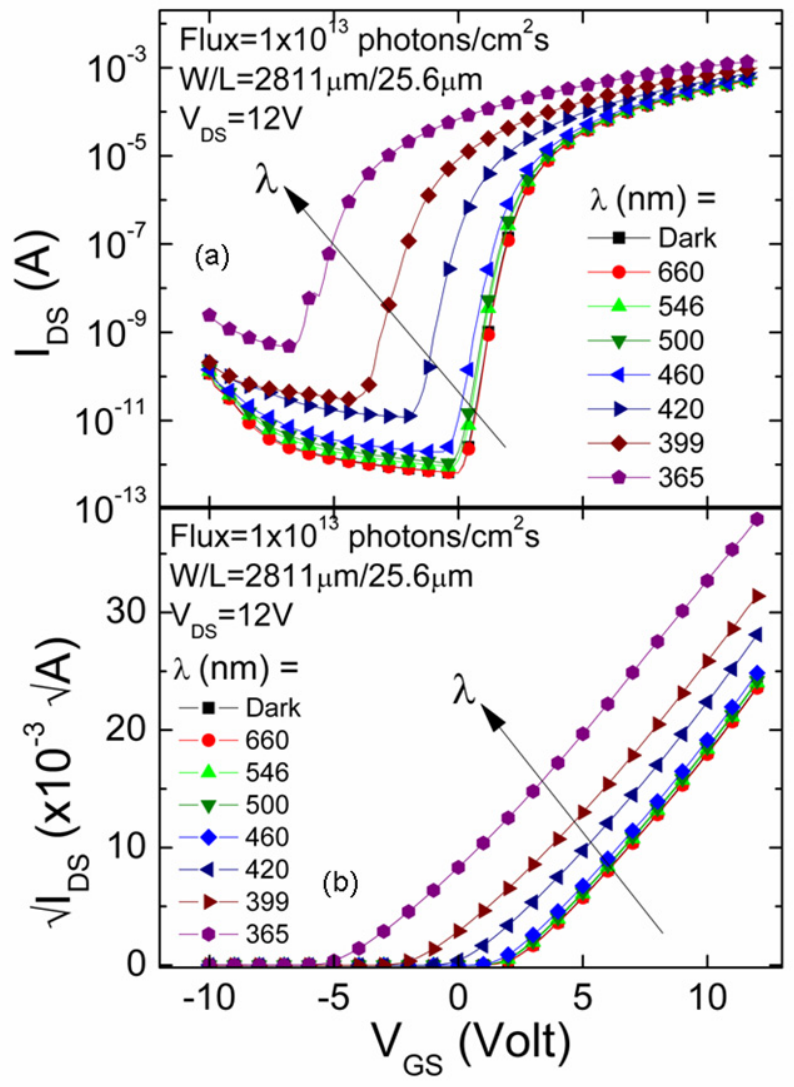

Figure 4. $I_{D S}-V_{G S}$ curves for constant photo flux with the varying light wavelengths.

\subsubsection{Under illumination}

The response of the PLD a-IGZO TFT to monochromatic illumination has been studied by measuring the TFT transfer characteristic for various light wavelengths. Fig. 4 shows the aIGZO TFT transfer characteristic in the dark and under illumination. We observed clearly a shift in TFT electrical properties under illumination. Furthermore, a "threshold" wavelength exists when TFTs are illuminated, Fig. 4. In other words, little or no shift occurs in TFT properties under illumination with $\lambda>420 \mathrm{~nm}(2.95 \mathrm{eV})$ while a much larger change takes place when $\lambda<420 \mathrm{~nm}$.

As shown in Fig. 4(a) under illumination, TFT $\mathrm{I}_{\mathrm{DS} \text {-off }}$ increases significantly as the illumination wavelength getting closer to UV region. Compare to the original dark condition, about three orders of magnitude increase occurs when we illuminated the device at

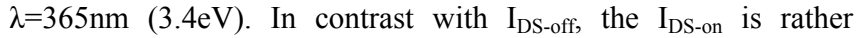
constant. The onset of off-to-on $\mathrm{V}_{\mathrm{GS}}$ voltage (or $\mathrm{V}_{\mathrm{ON}}$ ) also changes. Initially, the $\mathrm{V}_{\mathrm{ON}}$ is very close to zero volts for TFT in the dark and a negative shift in $V_{O N}$ is observed during illumination; $V_{O N} \sim-7$ volt was recorded for $\lambda=365 \mathrm{~nm}(3.4 \mathrm{eV})$. Finally, the off-to-on switching in $\mathrm{I}_{\mathrm{DS}}$ becomes less steep when we illuminate the TFT under shorter wavelength. As consequence, the TFT subthreshold swing increases. In on-region, a negative shift of $\mathrm{V}_{\mathrm{TH}}$, similar to $\mathrm{V}_{\mathrm{ON}}$ as shown in Fig. 4(b), is observed. The threshold voltage shift $\left(\Delta \mathrm{V}_{\mathrm{TH}}=\mathrm{V}_{\mathrm{TH} \text {-under illumination }}-\mathrm{V}_{\mathrm{TH} \text {-in dark }}\right)$ was determined to be about negative $6 \mathrm{~V}$ under illumination at $\lambda=365 \mathrm{~nm}(3.4 \mathrm{eV})$. The slope of $\sqrt{\mathrm{I}_{\mathrm{DS}}}-\mathrm{V}_{\mathrm{GS}}$ shows almost no change within the wavelength range used in this study (Fig. 4(b)). This suggests the field-effect mobility $\left(\mu_{\text {eff }}\right)$ is not affected by illumination.

\section{Discussion}

Fig. 5 shows the light transmittance spectra of LCD color filter [10] and light emission spectra of the AM-PLED [11]. Their maximum blue, green and red responses are about 450, 550 and $650 \mathrm{~nm}$, respectively.

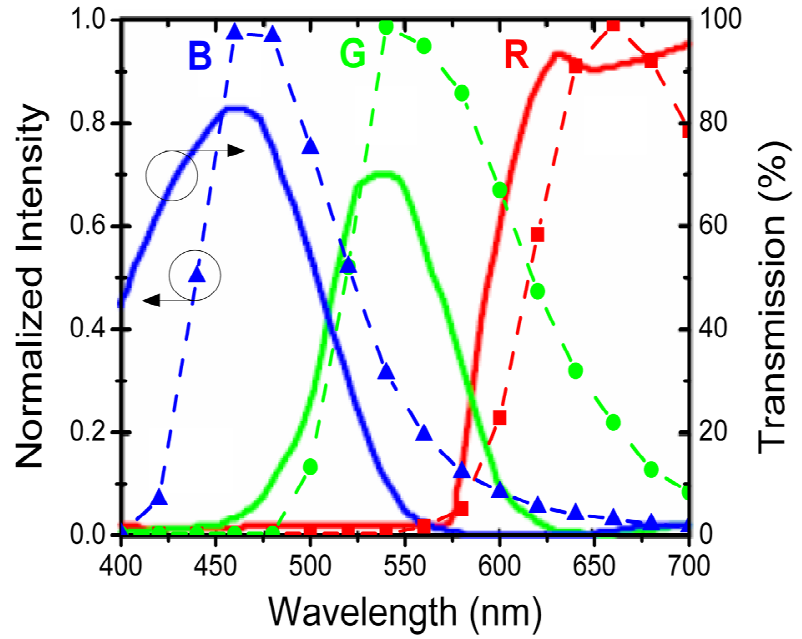

Figure 5. Example of the color filter transmission spectra of the LCD (solid curves) and light emission spectra from multicolor PLED (symbol and dash curves). 


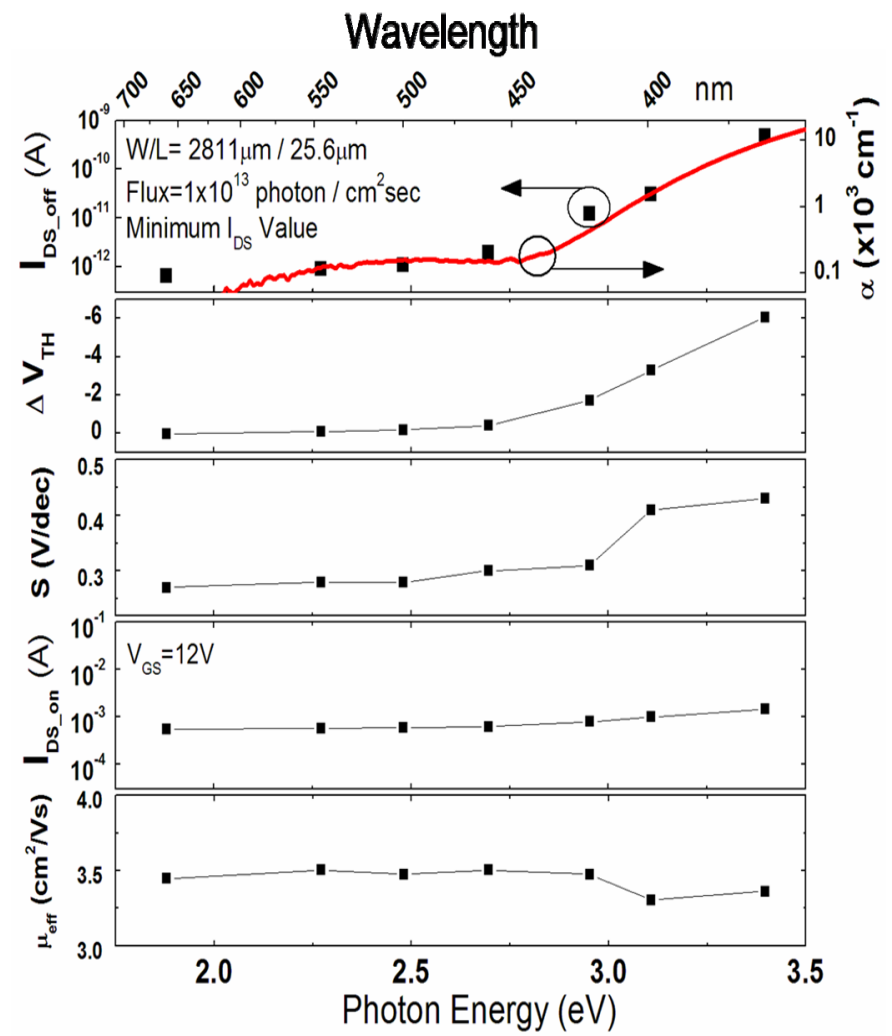

Figure 6. Dependence of PLD a-IGZO TFT minimum offstate drain current ( $\left.\mathrm{I}_{\mathrm{DS}-\mathrm{off}}\right)$, on-state drain current ( $\left.\mathrm{I}_{\mathrm{DS}-\mathrm{on}}\right)$, threshold voltage shift $\left(\Delta V_{\text {th }}\right)$, subthreshold swing (S), and field-effect mobility ( $\left.\mu_{\text {eff }}\right)$ on incident photons energy for constant photon flux.

From these figures, it is clear that the illumination wavelengths (365 660nm) in this experiment do cover the photon energy range of interest to AM-LCDs and AM-OLEDs, and the optical band-gap of the a-IGZO $(\sim 410 \mathrm{~nm})$. We summarize our results by plotting various TFT parameters as a function of incident photon energy (Fig. 6). By following (3) (5) and other definitions previously described in section 3.2, TFT off-state drain current ( $\mathrm{I}_{\mathrm{DS} \text { off }}$ ), threshold voltage shift $\left(\Delta \mathrm{V}_{\mathrm{th}}\right)$, subthreshold swing (S), on-state drain current ( $\left.\mathrm{I}_{\mathrm{DS} \_ \text {on }}\right)$ and $\mu_{\text {eff }}$ are extracted and shown in Fig. 6.

In the order to elucidate the physical origin of the shift in TFT electrical properties, the a-IGZO absorption spectrum (solid curve) is overlapped with the variation of the $\mathrm{I}_{\mathrm{DS}}$ off data in Fig. 6. It can be concluded that the light with energy less than $3.0 \mathrm{eV}$ (visible region) is only weakly absorbed and has a negligible effect on the TFT transfer characteristics, while light with energy larger than 3.0 $\mathrm{eV}$ is strongly absorbed and is expected to generate a large density of electron-hole pairs that are separated in the device channel. During illumination, the threshold voltage $\left(\mathrm{V}_{\mathrm{th}}\right)$ shifts to more negative $V_{G S}$ values with the increasing photo energy, indicating that the holes are trapped (positive space charge) within the channel or/and at the $\mathrm{SiO}_{2} /$ a-IGZO interface, e.g., electrons appears to be more mobile than holes in a-IGZO TFTs. The TFT $\mathrm{I}_{\mathrm{DS} \_ \text {off, }} \Delta \mathrm{V}_{\mathrm{th}}$, and subthreshold swing (S) are increasing with the increasing film absorption coefficient. After illumination, we can bring the device back to its original pre-illumination state by a $100^{\circ} \mathrm{C}$ thermal treatment for 3 minutes. With no applied heat, the device will regain its pre-illumination properties after a much linger period of time. Since this is a fully reversible process, the photogenerated carriers (holes) are most likely trapped during photo-generation process. The exact nature of the traps is under present investigation.

\section{Conclusion}

In conclusion, we have shown that the a-IGZO TFTs off-state drain current, subthreshold swing, and $\mathrm{V}_{\text {th }}$ shift under illumination follows material optical absorption spectrum. The a-IGZO TFT is stable under visible light illumination while electrical properties start to change when the illuminating photon energy is approaching or is above its absorption gap energy ( $\sim 3 \mathrm{eV}$, near-UV region) The illuminated devices can be return to their initial dark state after just short thermal treatment at higher temperature. This study indicates how the a-IGZO TFTs photosensitivity could affect active-matrix LCDs and OLEDs, and photo-sensors operation. May be light shield electrodes used in a-Si:H TFT AM-FPDs or photo-imagers could be avoided when a-IGZO TFTs are used?

\section{Acknowledgements}

All PLD films used in this work were deposited by Dr. K. Nomura at Tokyo Institute of Technology, Japan. All device fabrications and measurements were done at the University of Michigan, USA. Two of us (T.C. Fung and J. Kanicki) would like to thank DARPA for partial financial support.

\section{References}

[1] H. Hosono et al, "Working hypothesis to explore novel wide band gap electrically conducting amorphous oxides and examples" J. Non-Cryst. Solids, 198-200, 165-169, (1996).

[2] J. F. Wager, “Transparent electronics" Science, 300, 12451246, (2003).

[3] H. Hosono, "Ionic amorphous oxide semiconductors: material design, carrier transport, and device application" J. NonCryst. Solids, 352, 851-858, (2006).

[4] H. Hosono et al, "Room-temperature fabrication of transparent flexible thin film transistors using amorphous oxide semiconductors" Nature, 432, 488-492, (2004).

[5] K. Nomura et al, "Amorphous oxide semiconductor: materials, carrier transport and TFT characteristics" TAOS, Japan, (2006).

[6] T. Li et al, "Interference fringe-free transmission spectroscopy of amorphous thin films" J. Appl. Phys., 88, 5764-5771, (2000)

[7] J. Kanicki, Amorphous \& Microcrystalline Semiconductor Devices, Volume II: Materials and Device Physics, p.6, Boston: Artech House, (1992).

[8] R.A. Street, Hydrogenated Amorphous Silicon, p.88, Cambridge: Cambridge University Press, (1991).

[9] S. Sherman et al, "Correlation between the valence- and conduction-band tail energies in hydrogenated amorphous silicon” Appl. Phys. Lett., 69, 3242, (1996)

[10] M. Tani et al, "Progress in color filters for LCDs" Proc. IDRC , 103-111, (1994).

[11] J. Kanicki et al, "Optoelectronic properties of PLEDs on a plastic substrate” J. SID, 13/12, 993-1002, (2005) 